\title{
Monitoreo anestésico de la mezcla tiopental-propofol en hembras caninas durante la ovariohisterectomía
}

\author{
Anesthetic monitoring of the thiopental-propofol mixture in canine \\ females during ovariohysterectomy
}

\section{Monitorização anestésica que mistura tiopental-propofol em cadelas durante ovariohisterectomía}

\author{
Ángela C. León-Z ${ }^{1}$, Jorge M. Cruz-Amaya ${ }^{2 *}$ \\ MVZ Práctica privada. \\ 2 MV, PhD; Docente, Universidad Nacional de Colombia, Sede Medellín. Grupo de investigación en Biodiversidad y \\ Genética molecular (BIOGEM). \\ Email: jmcrusa@unal.edu.co
}

Recibido: 10 de diciembre de 2016

Aceptado: 25 de febrero de 2019

\begin{abstract}
Resumen
Objetivo: Monitorizar la fracción espiratoria final de $\mathrm{CO}_{2}$ (capnometría), saturación de oxígeno en la hemoglobina (pulsioxímetria) y la presión arterial media no invasiva (PAM) en perras sanas durante la ovariohisterectomía (OVH), en las cuales la anestesia fue inducida y mantenida con la mezcla Propofol-Tiopental (P-T). Materiales y métodos: Se practicó analítica prequirúrgica. La premedicación incluyo acepromacina, ketoprofeno y tramadol, la inducción anestésica se realizó con una mezcla en partes iguales de propofol $10 \%$ y tiopental $2.5 \%$. La dosis de inducción se calculó en base al tiopental presente en cada $\mathrm{cm}^{3}$ de la mezcla, y fue de $3 \mathrm{mg} / \mathrm{kg}$ de tiopental / IV. Se dieron bolos a efecto de $1 \mathrm{mg} / \mathrm{kg}$ de tiopental presente en la mezcla para el mantenimiento. La capnometría, pulsioximetría y PAM se midieron cada cinco minutos utilizando un monitor multiparametrico. Los datos se analizaron con un estudio inferencial destinado a encontrar un intervalo de confianza para la media de saturación de oxígeno en la hemoglobina $\left(\mathrm{SO}_{2}\right)$, fracción espiratoria final de CO2 $\left(\mathrm{FEFCO}_{2}\right)$ y PAM, con una confianza del 95\%. Resultados: $\mathrm{La} \mathrm{SO}_{2}$ mantuvo un promedio de 97\%, la PAM estuvo durante toda la intervención por encima de $60 \mathrm{mmHg}$, la FEFCO 2 fue alta inicialmente $>45 \mathrm{mmHg}$, pero retorno a valores normales luego de 35 minutos. En promedio no se presentó hipoxemia o hipotensión, pero si hipercapnia leve al comienzo. Conclusión: Para las tres variables estudiadas la mezcla P-T fue segura. Se sugieren otros estudios con un monitoreo más amplio para determinar la seguridad hemodinámica y respiratoria.
\end{abstract}

Palabras clave: Anestésicos parenterales; caninos; monitoreo anestésico.

\begin{abstract}
Abstrac
Objective: to monitor the final expiratory fraction of $\mathrm{CO}_{2}$ (capnometry), oxygen saturation in hemoglobin (pulse oximetry) and non-invasive mean arterial pressure (MAP) in healthy dogs during ovariohysterectomy $(\mathrm{OVH})$, in which anesthesia was induced and maintained with the Propofol-Thiopental (PT) mixture. Materials and methods: Pre-surgical analysis was performed. The premedication included acepromazine, ketoprofen and tramadol, the anesthetic induction was performed with a mixture in equal parts of propofol $10 \%$ and thiopental $2.5 \%$. The induction dose was calculated based on the thiopental
\end{abstract}


present in each $\mathrm{cm} 3$ of the mixture, and was $3 \mathrm{mg} / \mathrm{kg}$ of thiopental / IV. Bowls were given for the effect of $1 \mathrm{mg} / \mathrm{kg}$ of thiopental present in the mixture for maintenance. Capnometry, pulse oximetry and MAP were measured every five minutes using a multiparametric monitor. The data were analyzed with an inferential study aimed at finding a confidence interval for the mean oxygen saturation in hemoglobin $\left(\mathrm{SO}_{2}\right)$, final expiratory fraction of $\mathrm{CO}_{2}(\mathrm{FEFCO} 2)$ and PAM, with a confidence level of $95 \%$. Results: $\mathrm{SO}_{2}$ maintained an average of $97 \%$, MAP was throughout the intervention above $60 \mathrm{mmHg}, \mathrm{FEFCO} 2$ was initially high $>45 \mathrm{mmHg}$, but return to normal values after 25 minutes. On average there was no hypoxia or hypotension, but mild hypercapnia at the beginning. Conclusion: For the three variables studied, the P-T mixture was safe. Studies with broader monitoring are suggested to determine hemodynamic and respiratory safety.

Key words: Parenteral anesthetics; canine; anesthetic monitoring.

\section{Resumo}

Objectivo: para monitorar a fracção final de $\mathrm{CO}_{2}$ expiratória (capnometria), saturação de oxigénio na hemoglobina (oximetria de pulso) e pressão arterial média de forma não invasiva (PAM) em cães saudáveis durante ovariohysterectomy $(\mathrm{OVH})$, em que foi induzida a anestesia e mantida com a mistura Propofol-Thiopental (PT). Materiais e métodos: A análise pré-cirúrgica foi realizada. Acepromazina pré-medicação incluem, cetoprofeno e tramadol, a anestesia foi induzida com uma mistura de partes iguais de propofol e tiopental $10 \% 2,5 \%$. A dose de indução foi calculada com base no tiopental presente em cada $\mathrm{cm} 3$ da mistura e foi de $3 \mathrm{mg} / \mathrm{kg}$ de tiopental / IV. As taças foram dadas para o efeito de $1 \mathrm{mg} / \mathrm{kg}$ de tiopental presente na mistura para manutenção. A capnometria, a oximetria de pulso e a PAM foram medidas a cada cinco minutos usando um monitor multiparamétrico. Os dados foram analisados com um estudo inferencial para encontrar um intervalo de confiança para a saturação média de oxigénio da hemoglobina $\left(\mathrm{SO}_{2}\right), \mathrm{CO} 2$ final $(\mathrm{FEFCO})$ e fracção expiratório PAM, com 95\% de confiança. Resultados: $\mathrm{O} \mathrm{SO}_{2}$ mantida uma média de 97\%, MAP ao longo do processo era acima de $60 \mathrm{mmHg}$, a FEFCO 2 foi elevada inicialmente> $45 \mathrm{mmHg}$, mas voltam ao normal após 25 minutos. Em média, não houve hipoxemia ou hipotensão, mas hipercapnia leve no início. Conclusão: Para as três variáveis estudadas, a mistura P-T foi segura. Outros estudos com monitoramento mais amplo são sugeridos para determinar a segurança hemodinâmica e respiratória.

Palabras chave: Anestésicos parenterais; canino; monitorização anestésica.

\section{Introduccion}

En nuestro país es frecuente que la anestesia fija siga siendo muy utilizada en ciudades pequeñas y el campo, y seguramente este panorama permanecerá así por un tiempo debido al alto costo de las maquinas anestésicas.

Los anestésicos parenterales tienen múltiples ventajas y desventajas, con las cuales debe familiarizarse el clínico, existen pocos medicamentos de esta clase que ofrezcan un margen amplio de seguridad, siendo el costo y la accesibilidad en el mercado las limitantes más importantes para el uso de los fármacos seguros.

El tiopental y el propofol son anestésicos muy usados en la práctica clínica de manera independiente. El primero es un hipnótico eficaz y económico, indicado para la inducción de anestesia general (Plumb, 2010; Pawson y Forsyth, 2008). El segundo es un fármaco de acción ultracorta que provoca una inducción rápida y segura (Sumano y Ocampo 2016). El propofol parece activar los receptores del ácido y amino butírico (GABA) mediante su unión a un sitio diferente al tiopental, pero que determina la misma apertura de los canales de cloruro, causando híperpolarización celular (Sumano y Ocampo 2016). Este hecho hace suponer una posible interacción farmacológica al usarse mezclado con el tiopental.

El tiopental se acumula en el tejido adiposo y esto hace que su eliminación sea lenta, prolongando de forma inaceptable la recuperación de la anestesia si se suministran varios bolos (Riviere, 2011). De otra parte, con el propofol aun después de suministrar bolos repetidos la recuperación es rápida debido a su redistribución y metabolismo. Es de resaltar que ninguno de los dos posee propiedades analgésicas (Otero, 2012; Ko, 2012).

Las ventajas del propofol frente al tiopental son claras cuando se pretende mantener la anestesia con estos fármacos, pero al compararlos durante la inducción anestésica, no se han demostrado ventajas significativas del propofol (Niño, et al., 2007).

La combinación de P-T ha sido descrita en la práctica anestésica con pequeñas especies (Galindo, 2009; Calabor, 2008), al parecer reduce la posibilidad de contaminación bacteriana que el propofol presenta debido a sus excipientes que favorecen el crecimiento bacteriano y disminuye las desventajas del tiopental relacionadas con recuperación prolongada. 
La mezcla P-T posee un bajo costo en comparación con otros protocolos anestésicos, sin embargo han sido escasamente descritos sus efectos hemodinámicos y fisiológicos durante los procedimientos quirúrgicos rutinarios en caninos.

El objetivo de este trabajo fue monitorizar la fracción espiratoria final de $\mathrm{CO}_{2}$, La saturación de oxígeno en la hemoglobina y la presión arterial media en un grupo de perras sanas durante la ovariohisterectomía (OVH), en las cuales la anestesia fue inducida y mantenida con la mezcla P-T.

\section{Materiales y métodos}

Este proyecto fue aprobado por la comisión de ética para la experimentación animal de la Universidad de Antioquia (Acta $N^{\circ} 65$ ).

Se utilizaron 5 perras mestizas, con edades entre 1 y 5 años, y un peso inferior a $10 \mathrm{~kg}$, procedentes de una institución privada dedicada al bienestar animal. La anestesia fue inducida y mantenida con la mezcla de P-T, y posteriormente se practicó la OVH.

Previo a la intervención se realizó examen físico, clasificación según las normas de la Sociedad Americana de Anestesiólogos (ASA), y analítica pre- quirúrgica (hematocrito, creatinina, alanin amino transferasa y proteínas totales).

Los animales se premedicaron treinta minutos antes de la intervención con ampicilina (20 mg / kg / IV), tramadol (1 mg / kg / IM), acepromacina (0.05 mg / kg / IV) y ketoprofeno (1 mg / Kg / SC), se infundió Ringer lactato a una velocidad de $20 \mathrm{ml} / \mathrm{kg} /$ hora, durante todo el procedimiento quirúrgico.

El campo quirúrgico se preparó depilando la piel del abdomen y realizando la asepsia con jabón y solución de clorhexidina, posteriormente la anestesia se indujo con la mezcla P-T, dicha mezcla se realizó con partes iguales de ambos fármacos, cada centímetro de la mezcla contenía $12,5 \mathrm{mg}$ de tiopental y $5 \mathrm{mg}$ de propofol.

$\mathrm{Al}$ inducir la anestesia se introdujo una sonda endotraqueal para suministrar oxígeno y cuantificar la fracción espiratoria final de $\mathrm{CO}_{2}$.

El tiopental fue preparado en una dilución al 2.5\%; para la inducción se tuvo en cuenta la cantidad del tiopental presente en la mezcla, calculando $3 \mathrm{mg} / \mathrm{kg}$ del producto para inducir como guía inicial y bolos a efecto de $1 \mathrm{mg} / \mathrm{kg}$ para mantener la profundidad anestésica. Durante todo el procedimiento quirúrgico se tomaron las constantes vitales, además se midió la $\mathrm{SO}_{2}$, PAM y la FEFCO 2 cada cinco minutos, utilizando un monitor de marca Mindray PM-8000 exprees ${ }^{\circledR}$.

Para realizar la OVH se utilizó la técnica descrita por Fossum (2012), con abordaje al abdomen a través de la línea alba. Al terminar el procedimiento el paciente se vigiló para determinar el tiempo que tardó en ponerse en decúbito esternal desde la finalización de la cirugía. Además, se instauró un tratamiento antibiótico con ampicilina (20 mg / Kg / IM) cada 12 horas por 5 días, una terapia antiinflamatoria y analgésica con Ketoprofeno ( $1 \mathrm{mg} / \mathrm{Kg} / \mathrm{SC}$ ) cada 24 horas durante 3 días, acompañado de Tramadol (1 mg / Kg / IM) cada 12 horas durante 5 días, limpieza y desinfección de la herida quirúrgica dos veces al día.

\section{Análisis estadístico}

Se realizó un estudio inferencial donde se pretendió encontrar un intervalo de confianza para la media de $\mathrm{SO}_{2}$, PAM, y la FEFCO2, con una confianza del 95\%.

Para estimar el intervalo de confianza se debe precisar si la población se distribuye normalmente, se utilizó la prueba de Anderson Darling (AD), donde se asumió una confianza del 95\%.

Como criterio decisorio se tienen:

$H_{0}$ La variable $\left(\mathrm{SaO}_{2} ; \mathrm{FEFCO}_{2}, \mathrm{PAM}\right)$ sigue una distribución normal

$H_{a} \quad$ La variable $\left(\mathrm{SaO}_{2} ; \mathrm{FEFCO} 2, \mathrm{PAM}\right)$ no sigue una distri* bución normal.

\section{Resultados}

Con base en el examen físico, la anamnesis y la analítica pre quirúrgica se estableció un riesgo anestésico ASA I, para las cinco pacientes.

Todas las medias reportadas se analizaron a una misma concentración de P-T. Las intervenciones quirúrgicas tuvieron una duración promedio de 55 minutos con extremos de duración de 38-62 minutos.

En promedio se observó una $\mathrm{SO}_{2}$ de 97\% durante todas las intervenciones, con un rango de promedios de $96-98 \%$. A un nivel de significancia de 0.05, se puede concluir que la $\mathrm{SO}_{2}$ se distribuyó de forma normal, es decir que en promedio no disminuyó a niveles que indicaran hipoxemia (Grafico 1 y 2). Se considera que una $\mathrm{SO}_{2}$ inferior a 95\% es indicativa de dicha complicación. Una paciente (P3), presentó una disminución 


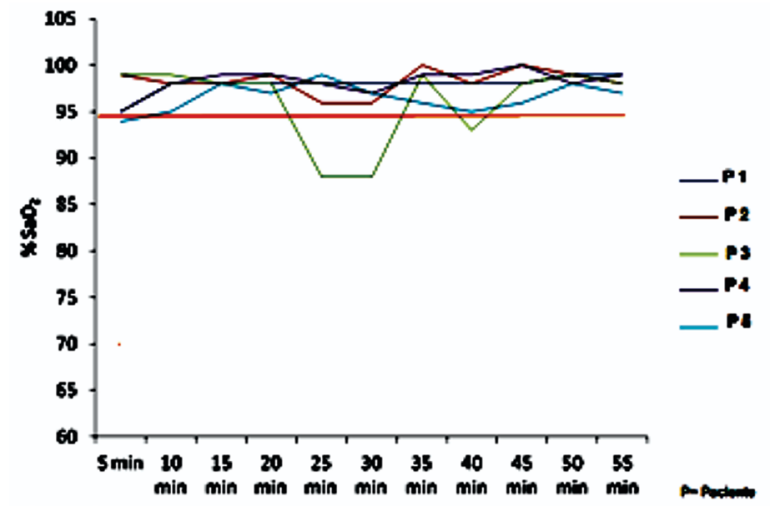

Grafico 1. Saturación de oxígeno en la hemoglobina, comportamiento de cada paciente (P1-P5) a través del tiempo. Los datos se distribuyen en promedio sobre el nivel inferior normal $\left(95 \% \mathrm{SO}_{2}\right)$.

importante de la $\mathrm{SO}_{2}$ durante un lapso de unos 10 minutos, pero luego se recuperó.

La PAM se mantuvo en todas las pacientes en valores superiores a $60 \mathrm{mmHg}$, límite que indica hipotensión (Grafico 3). A un nivel de significancia de 0.05 , se puede decir que la PAM se encuentra en un rango normal, sin disminuciones que indicaran hipotensión durante la intervención (Grafico 4). En dos pacientes (P1 y P2) se observó un aumento importante de la PAM, seguramente relacionado con anestesia superficial. Los valores de PAM durante la anestesia superiores $160 \mathrm{~mm}$ Hg deben hacer pensar en plano analgésico o hipnótico superficial.

El $\mathrm{CO}_{2}$ al inicio de la cirugía presentó en promedio valores altos respecto a sus rangos de referencia (35-45 $\mathrm{mm} \mathrm{Hg}$ ), lo que pudo ser causado durante la inducción anestésica ya que algunas perras presentaron apnea (Grafico 5). En este trabajo se observó que la $\mathrm{FEFCO}_{2}$ se mantuvo en niveles de $42.5 \mathrm{~mm} \mathrm{Hg}$ en promedio.

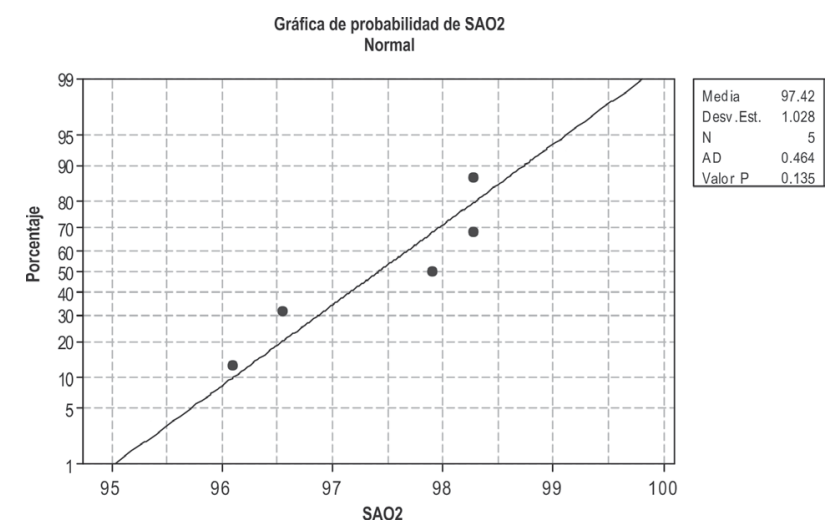

Grafico 2. Prueba de normalidad para SO2.

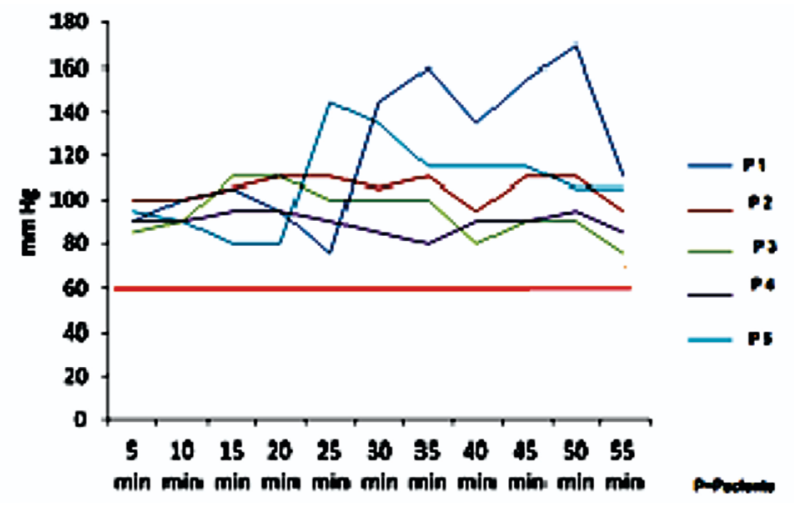

Grafico 3. Comportamiento de la PAM en cada paciente (P1P5) durante el tiempo. Los datos están sobre el límite inferior normal.

A un nivel de significancia de 0.05 , se puede decirse que la $\mathrm{FEFCO}_{2}$ se distribuye de forma normal, lo que indica que no se produjo acumulación importante de $\mathrm{CO}_{2}$ durante las intervenciones (Grafico 6).

La recuperación luego de la cirugía se produjo lentamente, en promedio las pacientes tardaron 84 minutos para adquirir el decúbito external y 208 minutos para caminar.

\section{Discusión}

Naguid y Sari (1991), describieron el efecto hipnótico de la mezcla P-T en humanos y concluyeron que dicha mezcla tenia efectos sinérgicos, si bien, la ventaja de utilizar esta combinación se conoce desde entonces, en anestesiología veterinaria las investigaciones con esta combinación son escasas.

En este trabajo se observó que la mezcla P-T utilizada en hembras caninas durante la $\mathrm{OVH}$ mantuvo dentro de límites estables las variables de PAM, $\mathrm{FEFCO}_{2}$ y $\mathrm{SO}_{2}$

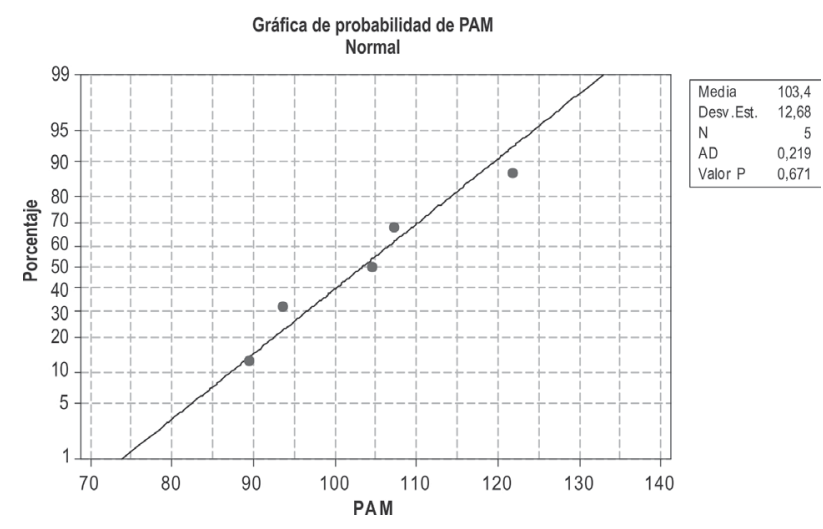

Grafico 4. Prueba de normalidad para PAM. 


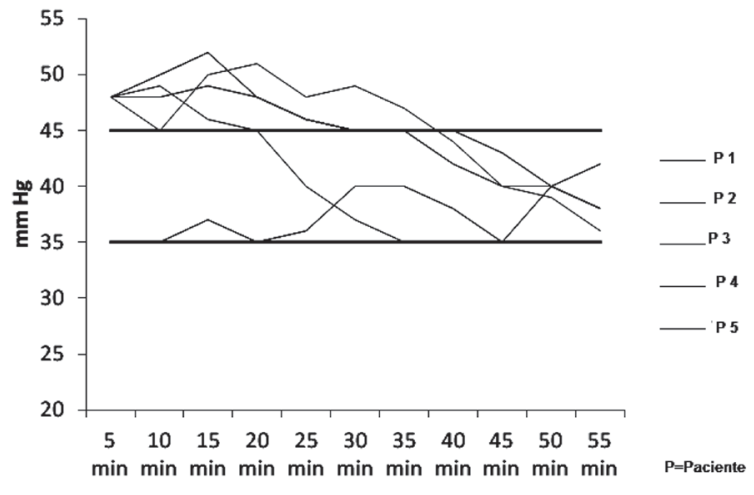

Grafico 5. Comportamiento de la $\mathrm{FEFCO}_{2}$ de cada paciente (P1-P5) a través del tiempo.

en promedio. Padilla y Cardona (2013), monitorizaron la PAM, y otras variables hemodinámicas durante la inducción y el mantenimiento de caninos sanos utilizando propofol, tiopental y la mezcla P-T en grupos diferentes, sin encontrar diferencias significativas en estos parámetros entre grupos.

Durante la anestesia general los valores de PAM, $\mathrm{SO}_{2}$ y $\mathrm{FEFCO}_{2}$ no deben ser inferiores a $60 \mathrm{~mm} \mathrm{Hg} \mathrm{y}$ $95 \%$ respectivamente para las dos primeras variables, mientras que para la tercera variable no debe superar 45 mm Hg (Muñoz et al., 2019, Grimm et al., 2011; Dugdale, 2010). Los anestésicos inducen depresión respiratoria, lo que suele producir elevaciones de la $\mathrm{FEFCO}_{2}$ y disminuciones de la $\mathrm{SO}_{2}$. Estos cambios se deben a diversas condiciones como: respuesta reducida de los quimiorreceptores centrales al $\mathrm{CO}_{2}$, disminución del volumen mínuto y desequilibrios de la ventilación / perfusión (Grimm et al., 2011; Muir, et al., 2012). En este estudio la $\mathrm{SO}_{2}$ presentó una media de $97 \%$ (Grafico 2). Valores inferiores a 95\% de $\mathrm{SO}_{2}$ indican hipoxemia (Ko 2012; Muir, et al., 2012). A la luz de estos hallazgos es posible considerar que la mezcla P-T es una alternativa, que no afecta la capacidad de oxigenar la sangre.

La $\mathrm{FEFCO}_{2}$ es uno los parámetros importantes a la hora de establecer si la ventilación es adecuada en un animal anestesiado. Se observó que la $\mathrm{FEFCO}_{2}$ se mantuvo en niveles de $42.5 \mathrm{~mm} \mathrm{Hg}$ en promedio. La $\mathrm{FEFCO}_{2}$ al inicio de la cirugía superó el límite de $45 \mathrm{~mm} \mathrm{Hg}$, debido a la apnea que se presentó en el momento de la inducción anestésica (tres pacientes), luego de intubar y suministrar oxígeno, los niveles de $\mathrm{CO}_{2}$ empezaron a normalizarse, evento que se observó hacia los 25 minutos de iniciado el procedimiento. Con frecuencia se observa apnea durante la inducción anestésica. Esta complicación se ha descrito de igual forma con el propofol o el tiopental cuando se utilizan

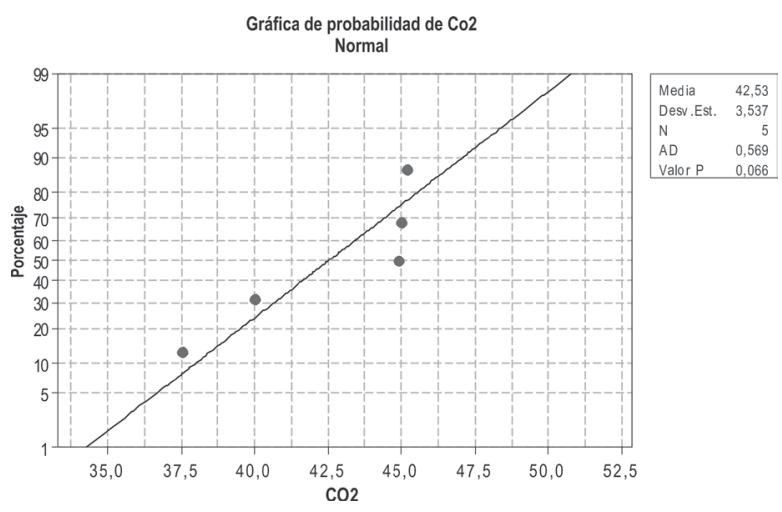

Grafico 6. Prueba de normalidad para $\mathrm{FEFCO}_{2}$.

de forma separada (Sumano y Ocampo, 2016). Una premedicación con un componente de analgesia y sedación más intensos, que utilice opiodes agonistas mu y tranquilizantes mayores (neuroleptoanalgesia) da la posibilidad de inducir con un bolo menor de la mezcla P-T y disminuye la aparición de apneas. Además, la dosis de inducción utilizada como guía de $3 \mathrm{mg} / \mathrm{kg}$ de tiopental, no fue efectiva para dicho propósito, requiriéndose una dosis mayor, esto hace pensar que se requiere una premedicación con sedación y analgesias más intensas para trabajar con la mezcla P-T.

La PAM es una variable utilizada en anestesiología para establecer la calidad de la circulación, ya que nos da una idea indirecta del gasto cardiaco (Muñoz, et al., 2019; Snyder y Johnson 2014). Se observó una PAM de $103 \mathrm{~mm} \mathrm{Hg}$ en promedio (grafico 4). La mezcla P-T produjo estabilidad cardiovascular, al menos para la variable PAM durante todo el procedimiento quirúrgico, no obstante, ambos fármacos pueden causar hipotensión cuando se utilizan de manera separada en el mantenimiento anestésico (Sumano y Ocampo, 2016). Debe considerarse que los fluidos infundidos durante la cirugía ayudaron de manera preventiva a evitar la hipotensión. Se eligió una dosis alta $(20 \mathrm{ml} /$ $\mathrm{kg} /$ hora), pensando en la posibilidad de sangrados y el efecto hipotensor que se conoce para ambos anestésicos. En una de las pacientes se observó un aumento importante de la PAM, seguramente relacionado a dolor y superficialidad en la anestesia. No debe olvidarse que ni el pentotal o el propofol son verdaderos analgésicos y que el método de suministro en bolos de la mezcla P-T no necesariamente mantiene niveles óptimos en plasma.

En la literatura se ha descrito que la recuperación de la anestesia con la mezcla P-T es notablemente mejor que con el tiopental solo (Galindo, 2009) y muy similar a la del propofol (Calabor, 2008), En este trabajo 
se observó que la recuperación de las pacientes tardo aproximadamente 84 minutos para que adquirieran el decúbito esternal y 208 para caminar, y si bien, esta recuperación puede considerarse muy prolongada si se compara con el isofluorano en el cual el decúbito esternal suele asumirse alrededor de los 20-30 minutos (Dugdale 2010; Muir, et al., 2012), es relativamente más corta que cuando se utiliza tiopental solo (6-8 horas), especialmente cuando se dieron muchos bolos para mantener la anestesia (Sumano y Ocampo, 2016), pero es más prolongada que al utilizar propofol. Al respecto los autores consideran que una premedicación con neuroleptoanalgesia podría disminuir el número de bolos para el mantenimiento y por lo tanto el tiempo de recuperación.

El propofol es un fármaco de fácil contaminación debido a sus excipientes (aceite de soja, lecitina de huevo), los fabricantes recomiendan descartar el sobrante después de 6 horas de abierto el recipiente y extremar las medidas asépticas durante su manipulación (Cabezas y Sandéz, 2014). Se reportó que el uso de propofol aumenta el riesgo de infección de la herida quirúrgica por lo menos 3.8 veces en cirugías limpias cuando se compara con animales que no recibieron el fármaco durante la anestesia (Snyder y Johson; 2014; Heldmann, et al., 1999). La adición de tiopental que presenta un $\mathrm{pH}$ muy alcalino podría disminuir las posibilidades de contaminación del propofol. Calabor (2008), describe estabilidad de la mezcla P-T de al menos una semana a temperatura ambiente.

\section{Conclusión}

Se observó un comportamiento aceptable en las variables evaluadas durante la anestesia con la combinación de P-T. Se recomienda realizar otros experimentos con esta mezcla aumentando el número de la muestra para determinar con más exactitud su seguridad.

\section{Bibliografía}

Calabor I. Inductores anestésicos en pequeños animales. (2008); [accedido en octubre, 2014]. URL:www.ucm.es.info/sevicema

Fossum T. 2012. Small Animal Surgery. 3 ed. St. Louis. Mosby. p745.

Galindo V. 2009.Guía práctica de anestesia en pequeños animales. Bogotá. LitoChalver S.A. p45.

Helmann E, et al. The association of propofol usage with postoperative wound infection rate in clean wounds: A retrospective study. Vet Surg. 1999; 28(4):256-259.

Muir W, Hubbell J, Skarda R, Bednarski R. 2012. Manual de Anestesia Veterinaria. 5 ed. Amsterdam. Mosby-Year Book. p234.

Muñoz P, Granados M, Navarrete R. 2019. Manuales clínicos de veterinaria. Anestesiología y cuidados intensivos. Barcelona, España: Elsevier. p. 22.

Naguib M, Sari K. Tiopentone-propofol hypnotic synergism in patients. Br J Anaesth. 1991;67(5):234-236.

Niño M, Chaves A, Salazar C. Cambios hemodinámicas durante la inducción anestésica con tiopental versus propofol en pacientes Asa I y II. Rev Colomb Anestesiol. 2007;(35):53-58.

Padilla C, Cardona R. Comparación de los efectos cardiovasculares del propofol, tiopental y de la mezcla propofol-tipental en un grupo de caninos sanos premedicados con hidromorfona. Rev Med Vet. 2013;(26):133-146.

Pandit, J. Intravenous anaesthetic agents. Anaesth Intensive Care. 2007;9(4):54-159.

Pawson P, Forsyth S. 2008. Agentes anestésicos. En: Madison J, Page J, Church, D. (Ed). Farmacología Clínica en Pequeños Animales. Buenos Aires, Argentina, Inter-Medica. p73 - 80.

Teppema J, Baby S. Anesthetics and control of breathing. Respir Physiol Neurobiol. 2011;(177):80-92.

Sumano H, Ocampo L. 2016. Farmacología Veterinaria; 3.ed. México: McGrawHill., p606. 